Revue d'histoire de l'Amérique française

REVUE D.HISTOIRE DE L'AMÉRIQUE FRANÇAISE

\title{
L'influence de la gauche catholique française sur l'idéologie politique de la CTCC-CSN de 1948 à 1964
}

\section{Simon Lapointe}

Volume 49, numéro 3, hiver 1996

URI : https://id.erudit.org/iderudit/305446ar

DOI : https://doi.org/10.7202/305446ar

Aller au sommaire du numéro

Éditeur(s)

Institut d'histoire de l'Amérique française

ISSN

0035-2357 (imprimé)

1492-1383 (numérique)

Découvrir la revue

Citer cet article

Lapointe, S. (1996). L'influence de la gauche catholique française sur l'idéologie politique de la CTCC-CSN de 1948 à 1964. Revue d'histoire de l'Amérique française, 49(3), 331-356. https://doi.org/10.7202/305446ar
Résumé de l'article

La Confédération des travailleurs catholiques du Canada (CTCC) connaît une importante mutation idéologique à la fin des années 1940. Ayant précédemment adhéré à une forme conservatrice de catholicisme social, la CTCC évolue vers un humanisme qui veut concilier catholicisme et libéralisme dans un esprit progressiste. Cette idéologie puise l'essentiel de ses sources dans le courant de pensée catholique de gauche français, en particulier chez les philosophes Emmanuel Mounier et Jacques Maritain. Tout en professant un catholicisme respectueux des dogmes, ceux-ci ont élaboré une pensée politique dont les grands axes sont la liberté de la personne, la justice sociale, la démocratie parlementaire, la laïcité et l'anti-nationalisme. C'est au nom de cette idéologie que la centrale syndicale déconfessionnalise ses structures et prend pour nom Confédération des syndicats nationaux (CSN) en 1960.
Tous droits réservés @ Institut d'histoire de l'Amérique française, 1996

Ce document est protégé par la loi sur le droit d'auteur. L'utilisation des services d’Érudit (y compris la reproduction) est assujettie à sa politique d'utilisation que vous pouvez consulter en ligne.

https://apropos.erudit.org/fr/usagers/politique-dutilisation/ 


\title{
L'INFLUENCE DE LA GAUCHE CATHOLIQUE FRANÇAISE SUR L'IDÉOLOGIE POLITIQUE DE LA CTCC-CSN DE 1948 À $1964^{1}$
}

\author{
SIMON LAPOINTE \\ Département d'histoire \\ Université d'Ottawa
}

\section{RÉSUMÉ}

La Confédération des travailleurs catholiques du Canada (CTCC) connaît une importante mutation idéologique à la fin des années 1940. Ayant précédemment adhéré à une forme conservatrice de catholicisme social, la CTCC évolue vers un humanisme qui veut concilier catholicisme et libéralisme dans un esprit progressiste. Cette idéologie puise l'essentiel de ses sources dans le courant de pensée catholique de gauche français, en particulier chez les philosophes Emmanuel Mounier et Jacques Maritain. Tout en professant un catholicisme respectueux des dogmes, ceux-ci ont élaboré une pensée politique dont les grands axes sont la liberté de la personne, la justice sociale, la démocratie parlementaire, la laïcité et l'anti-nationalisme. C'est au nom de cette idéologie que la centrale syndicale déconfessionnalise ses structures et prend pour nom Confédération des syndicats nationaux (CSN) en 1960.

\section{ABSTRACT}

At the end of the 1940s, the ideology of the "Confédération des travailleurs catholiques du Canada (CTCC)» underwent an important change. After having adhered to a conservative form of social Catholicism, the CTCC moved toward a humanism that attempted to reconcile Catholicism and liberalism in a progressive spirit. This ideology drew mainly on the thought of the French Catholic left, and notably on that of the philosophers Emmanuel Mounier and Jacques Maritain. While professing a Catholicism that respected the dogmas, these thinkers elaborated a political philosophy emphasizing personal freedom, social justice, parliamentary democracy, lay activity, and anti-nationalism. It was in the name of this ideology that the union deconfessionalized its structures and changed its name to Confederation of National Trade Unions (CNTU) in 1960.

1. Le présent article résume une partie de L'Influence de la gauche catholique française sur l'idéologie de la CTCC-CSN de 1948 à 1963, mémoire de maîtrise (histoire), Université de Montréal, 1995, 194 p.

RHAF, vol. 49, n 3, hiver 1996 
Le libéralisme catholique a été une des plus importantes voies d'entrée vers la modernité pour les intellectuels québécois. Pendant les années quarante et cinquante, les jeunes catholiques actifs au sein de Cité libre, du Devoir et de L'Action nationale ont opposé à l'idéologie clérico-conservatrice du régime duplessiste une pensée alliant foi catholique, démocratie et justice sociale ${ }^{2}$.

À cette époque, les syndicats de la Confédération des travailleurs catholiques du Canada (CTCC) $)^{3}$ participent eux aussi à ce mouvement intellectuel et se trouvent au centre de tous les combats et controverses menés contre le duplessisme. La centrale syndicale subit alors de profondes mutations idéologiques qui la font passer d'un conservatisme corporatiste à une idéologie qualifiée par Jacques Rouillard d'humanisme libéral ${ }^{4}$, tout en continuant de se réclamer des enseignements sociaux de l'Église.

Or il appert que certains catholiques ont suivi une démarche similaire en France, du début du siècle aux années cinquante. Qualifiés de catholiques de gauche, on les retrouve au sommaire de revues comme Sept, Esprit, La Vie intellectuelle, L'Aube et Ordre Nouveau; ils sont aussi membres de partis politiques comme le Parti démocratique populaire (PDP) et le Mouvement républicain populaire (MRP) ou militent au sein des syndicats de la Confédération française des travailleurs chrétiens (CFTC). On perçoit à la base de leur démarche un désir de concilier leurs convictions libérales et parfois même socialistes à leur foi catholique.

Le but de cet article est donc de montrer quels aspects de la pensée des catholiques de gauche français (en particulier celle des philosophes Jacques Maritain et Emmanuel Mounier) ont influencé l'idéologie politique de la centrale syndicale québécoise. Nous commencerons par présenter sommairement les catholiques de gauche français pour ensuite montrer dans quelles circonstances et par quels moyens la CTCC-CSN a adopté certains de leurs principes. Quelques éléments de l'idéologie politique de la centrale syndicale concernant la liberté de la personne, le bien commun, la démocratie, les relations Église-État et le nationalisme seront par la suite analysés pour identifier les éventuelles influences françaises.

2. Michael Behiels, Prelude to Quebec's Quiet Revolution (Montréal, McGill-Queen's University Press, 1985), 366 p.

3. Qui deviendra en 1960, à la suite de sa déconfessionnalisation, la Confédération des syndicats nationaux (CSN).

4. Jacques Rouillard, «Mutations de la Confédération des travailleurs catholiques du Canada (1940-1960)», Revue d'histoire de l'Amérique française, 34,3 (décembre 1980): 384. 


\section{I - LA GAUCHE CATHOLIQUE FRANCSAISE ET LA CTCC-CSN}

Par gauche catholique française, nous entendons ici surtout les intellectuels qui s'inscrivent dans la mouvance du Second Ralliement ${ }^{5}$, pendant les années 1920 et 1930 . Bien qu'ils soient partagés en plusieurs tendances souvent opposées ${ }^{6}$, il est possible de les classer grossièrement en deux grandes familles: les démocrates chrétiens et les personnalistes ${ }^{7}$.

Les démocrates chrétiens ${ }^{8}$, dont le représentant par excellence est le philosophe Jacques Maritain ${ }^{9}$, sont des catholiques fondamentalement libéraux, bien qu'ils se défendent d'adhérer au libéralisme doctrinaire condamné par Rome. Ils partagent avec les libéraux plus conventionnels plusieurs thèmes essentiels comme la démocratie parlementaire, la souveraineté du peuple, la liberté confessionnelle et les droits individuels. Ce libéralisme se double toutefois d'un catholicisme rigoureux et orthodoxe qui défend l'Église contre l'anticléricalisme.

Le groupe des personnalistes, dont le philosophe Emmanuel Mounier est la figure de proue, est encore plus hétérogène que celui des démocrates chrétiens. Il est composé des catholiques qui refusent l'ordre traditionaliste autant que l'ordre capitaliste et qui proposent une démarche qui dépasse de loin les intentions du Second Ralliement.

5. On désigne par Ralliements les deux mouvements qui ont conduit certains catholiques en France à accepter la république comme régime. Le Premier ralliement, déclenché en 1892 par l'encyclique Inter innumeras sollicitudines du pape Léon XIII, fait suite à l'échec du boulangisme. Quant au Second ralliement, il est une conséquence de la condamnation de L'Action française en 1926 par Pie XI.

6. Bien que l'appellation gauche catholique française soit utile pour nommer cette tendance idéologique, elle ne peut être qu'inexacte. Elle regroupe en fait une multitude de tendances concurrentes et parfois même opposées, se situant de la droite modérée à l'extrême-gauche du spectre idéologique français. Tous les auteurs s'étant penchés sur cette tendance s'entendent d'ailleurs pour en constater la «fragmentation extrême en partis, groupes et mouvements de toutes sortes». Voir Françoise Mayeur, L'aube; étude d'un journal d'opinion (1932-1940) (Paris, Armand Colin, 1966), 163.

7. Cette division, bien qu'elle soit arbitraire et nécessairement contestable, a toutefois été suggérée par plusieurs auteurs: Oscar L. Arnal, «Alternatives to the Third Republic Among Catholic Leftists in the 1930's», Historical Reflections/Réflexions historiques, 5 (hiver 1978): 177-194; François-Georges Dreyfus, Histoire de la démocratie française en France (Paris, Albin Michel, 1988), 431 p.; John Hellman, «The Opening to the Left in French Catholicism: the Role of the Personalists», Journal of the History of Ideas, 34,3 (juillet-septembre 1973): 381-391 et «French 'Left-Catholics' and Communism in the Nineteen Thirties», Church History, 45,4 (décembre 1976): 507-523.

8. Il faut souligner le caractère a posteriori de l'étiquette démocrate-chrétienne puisqu'aucun de ces groupes ne s'en est publiquement réclamé. On explique cette prudence par un désir d'éviter les foudres de Rome.

9. Sur l'influence de Maritain au Québec, voir Vianney Décarie, «Jacques Maritain», Le Devoir, 3 mai 1973, 4. 
Actifs au sein des revues Esprit, et Ordre nouveau et chez les prêtresouvriers, les personnalistes ont en commun la même révolte, le même refus d'une société qu'ils considèrent corrompue, sans âme. Leur pensée partage les mêmes thèmes, comme le refus du «désordre établi», l'importance de la personne, l'esprit communautaire et le christianisme comme source de renouveau révolutionnaire ${ }^{10}$.

L'influence des idées de Mounier et de Maritain commencent à être perceptibles à la CTCC vers la fin des années quarante. Après l'échec de la grève de l'amiante de 1949, causée en grande partie par le refus des patrons d'accepter les principes de cogestion et de copropriété, les militants de la CTCC remettent en question la théorie de la réforme de l'entreprise ${ }^{11}$. Ce remue-ménage idéologique a lieu alors que la centrale connaît une période où le recrutement de nouveaux membres devient de plus en plus problématique. À partir de 1954, alors que le mouvement syndical québécois poursuit sa période d'expansion d'après-guerre de façon plus ou moins constante, les effectifs de la centrale se mettent à stagner ${ }^{12}$. Toutes ces difficultés ont eu une influence certaine sur les militants, les incitant à réviser leurs conceptions idéologiques et à abandonner les structures confessionnelles de la centrale.

L'adoption par la centrale syndicale d'une nouvelle interprétation philosophique du catholicisme, nommée ici humanisme libéral, s'est essentiellement faite dans la continuité. Jusqu'à la déconfessionnalisation de ses structures en 1960, la CTCC oriente officiellement son action selon les principes de la doctrine sociale de l'Église; du corporatisme à la théorie de la réforme de l'entreprise, l'évolution idéologique de la centrale se fait à partir du même corpus doctrinal ${ }^{13}$. Avec l'humanisme libéral, la CTCC-CSN conserve la doctrine sociale de l'Église comme première référence, mais la réinterprète en laissant de côté ses aspects les plus conservateurs et en mettant de l'avant les idées plus progressistes. L'humanisme libéral ne se veut pas une forme hétérodoxe ou déviante du catholicisme. Au contraire, ses militants estiment plutôt, à l'instar de l'aumônier Jean-Marie Lafontaine, que:

10. Sur l'influence de la gauche catholique au Québec, voir Peter Taaffe, «The Influence of the French Catholic Left on Quebec 1945-1955», The Register, 4,1 (mars 1983): 53-72.

11. Rouillard, «Mutations...», op. cit., 383.

12. Si on considère les effectifs relatifs, on constate que la période est marquée par une diminution du pourcentage de syndiqués québécois membres de la CTCC. Atteignant un sommet de $35 \%$ en 1954, ce pourcentage descend jusqu'à $24,6 \%$ en 1961, le plus bas taux depuis la fin de la guerre. Voir Fernand Harvey, dir., Le mouvement ouvrier au Québec (Montréal, Boréal Express, 1980), 281-289.

13. Jean Sexton, La CTCC-CSN: du corporatisme à la réforme de l'entreprise, mémoire de maîtrise (relations industrielles), Université Laval, 1968, 93. 
de par sa nature, le christianisme n'est pas «conservateur». Il demande constamment que les institutions se renouvellent et s'adaptent; alors que nous sommes trop portés à nous satisfaire de ce qui a pu être utile dans le passé, le christianisme pousse à des transformations profondes dans les personnes et dans la société ${ }^{14}$.

Les militants humanistes-libéraux ne s'estiment pas progressistes malgré leur catholicisme mais plutôt à cause de leur catholicisme. Leur pensée prend sa source dans une réinterprétation des principes sociaux de l'Église romaine, proposant une nouvelle vision de l'être humain en tant que personne insérée dans une communauté naturelle.

C'est pourquoi les militants de la CTCC ne cessent de dénoncer les «mauvaises interprétations» des catholiques conservateurs qui, pour eux, sont nécessairement dans l'erreur. L'humanisme libéral est ainsi le fruit de deux désirs opposés mais complémentaires des militants: celui de réconcilier leur foi avec leurs idéaux de liberté et celui de marquer une distance face aux catholiques conservateurs.

C'est essentiellement avec l'arrivée de nouveaux permanents au sein des fédérations, des conseils centraux et de l'exécutif de la CTCC que la centrale adopte les principes fondamentaux de la gauche catholique française et s'oriente vers l'humanisme libéral ${ }^{15}$. Pour Gérard Pelletier, l'apport de «sang neuf» de la fin des années quarante est un événement décisif pour la CTCC:

Avec l'arrivée du tandem Picard-Marchand et de toute une équipe de militants nouveaux qu'ils entraînèrent à leur suite, la situation devait changer très rapidement. Si bien qu'au sortir de la guerre, la CTCC était devenue une centrale authentique, prête à relever les défis auxquels la classe ouvrière devait faire face au Québec ${ }^{16}$.

L'élection de Gérard Picard à la présidence de la centrale contre l'ancien président Alfred Charpentier, lors du congrès de 1946 (il a alors 39 ans), vient marquer symboliquement le besoin de renouveau de la CTCC. Picard fait son entrée à l'exécutif comme secrétaire trésorier en 1934, puis est élu secrétaire général en 1936. L'autre forte personnalité qui marque la centrale à cette époque est Jean Marchand. Diplômé de l'École des sciences sociales de l'Université Laval en 1942, il est engagé la même année comme organisateur syndical à la

14. Jean-Marie Lafontaine, «Le christianisme nous pousse à désirer des changements profonds", Le Travail, novembre 1963, 7. (Le Travail est l'organe officiel de la CTCC-CSN).

15. Plus de la moitié des membres du bureau confédéral sont des nouveaux venus en 1949. Jacques Rouillard, L'histoire de la CSN (1921-1981) (Montréal, Boréal-CSN, 1981), 169.

16. Gérard Pelletier, Les années d'impatience, 1950-1960 (Montréal, Stanké, 1983), 31. 
Fédération de la pulpe et du papier de la CTCC. Élu secrétaire général en septembre 1948, Marchand accepte également pendant deux ans la direction du journal interne de la CTCC (Le Travail), où il publie plusieurs articles et éditoriaux. Bien que Marchand soit avant tout un militant se consacrant à l'action, sa réflexion marque profondément l'idéologie de la centrale: il figure d'ailleurs parmi les auteurs des déclarations de principes de 1951 et de 1960. Après avoir activement milité en faveur de la déconfessionnalisation, il occupe la présidence de la centrale de 1961 à 1965, date où il joint les rangs du Parti libéral fédéral en compagnie de Pierre Elliott Trudeau et de Gérard Pelletier.

Autour du noyau Picard-Marchand se bâtit une équipe de militants qui ont suivi un parcours intellectuel similaire. Par exemple, Gérard Pelletier travaillait au Devoir comme reporter depuis l'arrivée de Gérard Fillion à sa direction en 1947 lorsqu'il fut mandaté par le journal pour couvrir la grève de l'amiante. Il avait précédemment milité, pendant ses études à l'Université Laval, comme secrétaire national de la Jeunesse étudiante catholique (JEC) de 1939 à 1943, puis comme secrétaire itinérant du Fonds mondial de secours aux étudiants, rattaché au secrétariat de l'organisation à Genève. C'est à cette occasion qu'il se lie d'amitié avec Jean Marchand qui lui cède la direction du Travail. Pelletier occupe ce poste par intermittence de 1950 à 1961. Pendant cette période, en plus de diriger la revue Cité libre dont il est un des cofondateurs, Pelletier travaille comme éditorialiste au journal syndical qu'il marque par son style rigoureux et incisif.

On assiste également à un renouvellement de personnel chez les aumôniers de la centrale ${ }^{17}$. En plus de leurs études en théologie, Henri Pichette, aumônier général de la centrale de 1945 à 1968, et PaulÉmile Bolté, aumônier général adjoint de 1953 à 1955, ont tous deux fréquenté l'École des sciences sociales de l'Université Laval. De façon générale, ces membres du clergé partagent avec enthousiasme les orientations idéologiques des permanents laïques de la centrale. En tant que membres de la Commission sacerdotale d'études sociales (CSES), ils jouent, durant les années cinquante, à la fois un rôle d'intermédiaire entre la CTCC et la hiérarchie catholique et un rôle d'orientation intellectuelle ${ }^{18}$.

Quelle que soit la position que ces militants occupent au sein de la centrale, leurs parcours intellectuels ont plusieurs points en com-

17. Hélène Bois, Les aumôniers et la déconfessionnalisation des institutions économicosociales québécoises (1940-1972), thèse de doctorat (histoire), Université Laval, 1991, 100-112.

18. Jacques Cousineau, L'Église d'ici et le social. 1940-1960 (Montréal, Bellarmin, 1982), 112. 
mun. Ils sont premièrement tous de la «génération des années 30» et furent, de ce fait, marqués par la Crise et la remise en question du capitalisme $^{19}$. Ayant été en contact avec l'idéologie du clergé catholique, ils ont pour la plupart développé une attitude critique face au nationalisme et aux valeurs traditionnelles. La guerre est venue par la suite confirmer leur hostilité au conservatisme, ayant assisté à la défaite des idéologies autoritaires droitistes.

La plupart d'entre eux ont débuté leur vie militante au sein des groupes d'action catholique spécialisée comme la JEC et la JOC. Ces premières années d'engagement politique leur donnent le goût de l'action concrète et les mettent en contact avec les principaux principes du libéralisme catholique: indépendance vis-à-vis le clergé, libertés individuelles, démocratie. Cet engagement s'est poursuivi chez certains comme Gérard Pelletier, Pierre Vadeboncoeur, Jean-Paul Geoffroy et Jean-Paul Lefebvre, au sein de la revue de Cité Libre ${ }^{20}$. Pierre Elliott Trudeau, cofondateur de la revue, travaille lui aussi ponctuellement pour la CTCC en donnant des cours de formation syndicale. Après la Révolution tranquille, plusieurs se lancent dans la politique active soit auprès du Parti libéral fédéral (Pelletier et Marchand), du NPD et des mouvements socialistes indépendantistes (Vadeboncoeur, Chartrand) ou du Parti québécois (Jean-Paul Lefebvre).

C'est donc principalement par ces nouveaux permanents syndicaux que la CTCC est exposée à l'influence de la gauche catholique française. Ceux-ci ont généralement été mis en contact avec ce courant d'idées par le biais de leurs études. Bien que la plupart des enseignants des collèges classiques et des universités leur aient dispensé un enseignement conforme à l'idéologie conservatrice du clergé, certains, comme François Hertel $^{21}$ et Georges-Henri Lévesque ${ }^{22}$, les ont initiés aux ouvrages essentiels du catholicisme de gauche.

19. Le personnalisme québécois commence à se développer au sein de la revue $L a$ Relève. Voir André-J. Bélanger, Ruptures et constantes, quatre idéologies du Québec en éclatement: La Relève, la JEC, Cité libre, Parti pris (Montréal, Hurtubise HMH, 1977), 33. De tous ses collaborateurs, c'est Guy Frégault qui élabore de façon systématique son adhésion au catholicisme de gauche; selon Christian Roy, il correspond régulièrement, dès la fondation de $L a$ Relève, avec Alexandre Marc, et devient l'animateur de ce qui se voulait la cellule canadienne de la revue personnaliste L'Ordre Nouveau. Voir Christian Roy, «Le personnalisme de L'Ordre Nouveau et le Québec, 1930-1947», RHAF, 46,3 (hiver 1993): 463-484.

20. Sur les relations entre l'équipe de Cité libre et celle de la revue Esprit, voir Lee S. Rotherham, "Quebec's Loudhailers: a Conflict of Nationality in the Journal Esprit», British Journal of Canadian Studies, 8,2 (1993): 191-210.

21. Le jésuite François Hertel élabore les postulats de base du catholicisme de gauche dans son ouvrage Pour un ordre personnaliste, paru en 1942. La pensée d'Hertel prend sa source dans les œuvres d'Arnaud Dandieu, de Nicolas Berdiaeff et de Jacques Maritain. Voir Jean Tétreau, Hertel, l'homme et l'œuvre (Montréal, Éditions Pierre Tisseyre, 1986), 78.

22. Dès sa fondation en 1938, l'École des sciences sociales de l'Université Laval, dirigée par le père Georges-Henri Lévesque, joue un rôle de «courroie de transmission» intellectuelle 
La guerre offre une autre occasion de contact entre les catholiques québécois et français. Par exemple, Jacques Maritain, exilé lors de l'occupation nazie, visite le Canada à plusieurs reprises pour des séries de conférences. Après la guerre, nombreux sont les penseurs catholiques de gauche qui sont engagés comme professeurs ou comme conférenciers dans les universités québécoises. Selon Gérard Pelletier, ces visites ont eu une importance capitale pour les intellectuels de sa génération: «Nous nous sommes imbibés du personnalisme en lisant Mounier, Landsberg et leurs collaborateurs. De plus, nous recevions la visite de deux compagnons de lutte de Mounier, Paul Vignaux et Henri Marrou, qui venaient tous deux enseigner à l'Institut d'études médiévales de l'Université de Montréal ${ }^{23} . »$

Pour certains militants $\mathrm{s}^{24}$, la prise de contact avec la gauche catholique se fait à l'occasion d'un voyage en France. Comme les principales revues de réflexion politique se rendaient de façon irrégulière au Québec, le séjour en France devient pour plusieurs une occasion de prendre littéralement un «bain» de personnalisme. Toujours selon Pelletier:

L'un de nos premiers soucis, en arrivant en France, fut d'approcher la revue et le mouvement Esprit. Avec quelles précautions toutefois et quel respect! Je me reproche encore, pour ma part, la timidité absurde qui me retint alors de me rendre carrément rue Jacob rencontrer Mounier, l'un des hommes du monde que j'admirais le plus. [...] Je me contentai d'aller l'entendre en conférence, de lire religieusement tout ce qu'il publiait, et d'approcher certains de ses jeunes collaborateurs. Heureusement, d'autres [...] ne furent pas victimes de cette révérence outrée ${ }^{25}$.

Finalement, un lien organique s'établit entre la CTCC-CSN et la gauche catholique française en 1947 quand la centrale s'affilie à la Confédération internationale des syndicats chrétiens (CISC). Fondée en 1921, l'orientation idéologique de cette organisation est influencée

entre les catholiques de gauche français et le Québec. L'École engage, tant par désir d'ouverture intellectuelle que par nécessité, des professeurs et des conférenciers étrangers comme Jacques Maritain et le père Delos d'Économie et Humanisme. Voir Georges-Henri Lévesque, «La première décennie de la Faculté des Sciences sociales à l'Université Laval», Colloque de la Société royale du Canada (1981), 11.

23. Gérard Pelletier, «Introduction», Yvan Lamonde et Gérard Pelletier, dir., Cité Libre: une anthologie (Montréal, Stanké, 1991), 10.

24. Un des cas typiques est celui de Guy Cormier, rédacteur du journal jociste Front Ouvrier puis «citélibriste». Parti en voyage d'études en France en 1951, il publie dans Le Travail une série d'articles portant sur la CFTC, le MRP et les prêtres ouvriers («Un lecteur voyage», Le Travail, 29 novembre 1951, 9).

25. Pelletier, Les années..., op. cit., 144. 
principalement par la Confédération française des travailleurs chrétiens. Après une longue période d'hésitation, la centrale finit par se joindre à l'organisation internationale, et c'est en grande partie par cet intermédiaire qu'elle prend contact avec Gaston Tessier et Paul Vignaux, les deux principaux théoriciens du syndicalisme catholique de gauche en France ${ }^{26}$.

L'influence du catholicisme de gauche atteint son apogée en 1960, alors que la CTCC déconfessionnalise ses structures et prend le nom de Confédération des syndicats nationaux. La centrale modifie au même moment sa déclaration de principes qui loge alors entièrement à l'enseigne de l'humanisme libéral ${ }^{27}$. Cette période prend fin en 1966, alors que Jean Marchand quitte la présidence de la centrale qui amorce alors un virage idéologique vers le socialisme démocratique ${ }^{28}$.

\section{2 - LA PERSONNE ET LA LIBERTÉ}

À la base de la philosophie politique de l'humanisme libéral de la CTCC-CSN se trouve la personne humaine. À l'instar de Jacques Maritain, pour qui «l'unité sociale est la personne ${ }^{29}$ », les militants de la centrale considèrent que c'est l'être humain qui est la mesure de toute chose dans le domaine temporel.

De plus, la personne se caractérise pour les humanistes-libéraux par sa faculté d'être libre. Alors que les catholiques de droite, faisant toujours passer la préservation sociale devant la promotion des droits de la personne, veulent limiter la liberté personnelle à cause de la faiblesse de la nature humaine, l'homme est considéré par les militants de la CTCC-CSN comme un être libre et autonome. Dans sa déclaration de principes de 1960, la centrale justifie la liberté de la personne par le fait que l'homme, créé à l'image de Dieu, est «doué d'intelligence et de volonté libre et qu'il a une destinée éternelle et surnaturelle. L'homme est donc un être personnel responsable de ses actes et de sa vie ${ }^{30} \gg$. Cette nouvelle approche de cette conception a pour conséquence de donner une importance de premier plan à la promotion des droits et libertés de la personne. Toujours dans sa

26. Hervé Hamon et Patrick Rotman, La deuxième gauche. Histoire intellectuelle et politique de la CFDT (Paris, Seuil, 1982), $456 \mathrm{p}$.

27. «Déclaration de principes de la CSN», Procès-verbal de la trente-neuvième session du congrès de la CSN (Montréal, 1960), 131-138. 342 .

28. Jacques Rouillard, Histoire du syndicalisme québécois (Montréal, Boréal, 1989), 336-

29. Jacques Maritain, «La personne et le bien commun», Oeuvres complètes (Fribourg, Éditions universitaires, 1990), IX: 198.

30. CSN, «Déclaration de principes de 1960...», 131. 
déclaration de principes de 1960 , la centrale lie la liberté de la personne à des droits spécifiques découlant de sa nature spirituelle: «[...] pour lui permettre d'accomplir sa destinée et de s'acquitter de ses obligations, l'homme est investi de droits naturels inaliénables qu'on ne peut jamais nier, abolir ou ignorer ${ }^{31}$.»

L'intérêt porté aux droits individuels se manifeste de façon récurrente tout au long des années cinquante et soixante. En 1954, le Congrès confédéral forme, par exemple, un comité chargé de rédiger une charte des droits que la centrale souhaite faire enchâsser dans la constitution canadienne ${ }^{32}$. La centrale publie également en 1963, dans son journal, le texte intégral de la déclaration universelle des droits de l'homme de l'ONU, qu'elle propose comme balise pour une future charte canadienne ${ }^{33}$. La déclaration de principes de 1960 contient d'ailleurs une déclaration de droits fondamentaux qui inclut:

1 - le droit au travail; 2 - le droit d'association; 3 - le droit à une juste répartition des richesses; 4 - le droit au respect de la personne dans sa vie physique et morale; 5 - le droit à la vérité, à l'information et à la culture; 6 - le droit à la sécurité juridique; 7 - le droit de s'exprimer librement; 8 - le droit de participer à la vie économique, sociale et politique de la nation ${ }^{34}$.

Cette nouvelle orientation idéologique est inspirée des penseurs catholiques de gauche français. Malgré la diversité de leur pensée, ceux-ci partagent la même conception de la personne, qui est la base de leur philosophie.

Comme la plupart des catholiques de gauche, Emmanuel Mounier ne propose pas de définition précise de ce qu'est une personne humaine, principalement parce que sa dimension mystique et subjective échappe aux analyses rationnelles. Pour lui, la personne est un centre inaliénable de décision et d'action, perçue à la fois comme un idéal, un absolu et comme une exigence ${ }^{35}$. De plus, il la considère comme une unité qui englobe à la fois l'individu, c'est-à-dire le corps, l'aspect matériel, et l'âme, l'aspect spirituel. Finalement, Mounier caractérise la personne par son autonomie, son libre-arbitre, sa capacité de choisir rationnellement. Bien qu'il soit un être naturel par son corps, l'homme se singularise, selon lui, «par une double capacité de rompre avec la nature. Seul il connaît cet univers qui l'engloutit, et

31. Ibid., 132 .

32. «Les droits de la personne humaine», Le Travail, 24 septembre 1954, 4.

33. «La déclaration universelle des droits de l'homme», Le Travail, décembre 1963, 10.

34. CSN, «Déclaration de principes de 1960...», 132.

35. Michel Barlow, Le socialisme d'Emmanuel Mounier (Toulouse, Privat, 1971), 72. 
seul, il le transforme ${ }^{36} \gg$. Profondément immergé dans sa nature matérielle, l'homme n'en subit pas pour autant la domination de façon inévitable. La personne échappe pour une grande part au déterminisme de la nature. Selon Jacques Maritain, «la nature n'a son visage en nous qu'accomplie par l'esprit, l'homme n'a sa vérité que modelée du dedans par la raison et la vertu ${ }^{37}$ ». La liberté, étant liée à la part divine de l'homme, est constitutive de la personne. Suivant cette logique, les droits de l'homme sont inaliénables pour Maritain, puisqu'ils sont «fondés sur la nature même de l'homme, que nul homme assurément ne peut perdre ${ }^{38}{ }$.

\section{3 - LIBERTÉ ET BIEN COMMUN}

Bien qu'elle revête une valeur fondamentale dans son idéologie, la CTCC-CSN ne pose toutefois pas la liberté personnelle comme un absolu. Selon les militants humanistes-libéraux, le personnalisme de la centrale n'a rien à voir avec l'individualisme matérialiste du libéralisme classique ou de l'anarchisme. Qualifié de «principe de destruction ${ }^{39}{ }^{\prime}$, l'égoïsme individualiste va à l'encontre de la nature même de la personne.

L'idéal communautaire porté par l'humanisme libéral se veut une synthèse entre la liberté individuelle et la promotion du bien commun. La liberté s'accompagne donc nécessairement de responsabilités. Pour la centrale, l'idéal communautaire vers lequel tout système politique doit tendre place l'être humain et sa liberté au premier plan, tout en établissant une relation d'ordre symbiotique librement consentie entre la personne et la société, l'une nourrissant et enrichissant l'autre dans un esprit de communion. Dans la déclaration de principes de 1960, on peut lire:

Comme sa nature l'exige, l'homme doit vivre en société pour développer ses facultés et réaliser sa destinée. Il doit être considéré comme le sujet actif de la vie sociale et non comme un simple objet. C'est un devoir pour chaque citoyen de contribuer au bien commun selon les exigences de la justice sociale ${ }^{40}$.

Les militants humanistes libéraux de la centrale semblent donc adhérer au principe voulant que «la liberté de l'un s'arrête où commence celle

36. Emmanuel Mounier, «Le personnalisme», Euvres (Paris, Seuil, 1962), III: 443.

37. Jacques Maritain, «Religion et culture», Euvres complètes (Fribourg, Éditions universitaires, 1983), IV: 200.

38. Idem, «L'homme et l'État», Euvres complètes, op. cit., IX: 597.

39. Philippe Bergeron, «Pour devenir un vrai syndiqué, il faut cultiver en soi le sens social et fixer l'objectif commun», Le Travail, décembre 1949, 15.

40. CSN, «Déclaration de principes de 1960...», 133. 
de l'autre», favorisant ainsi un modèle de société où la cohésion de la collectivité est basée sur un équilibre des libertés.

Les catholiques de gauche français ont longuement élaboré sur le thème de la conciliation de la liberté personnelle et des intérêts collectifs. Pour Mounier, une société véritablement chrétienne doit être personnaliste et communautaire. Bien que la personne soit en quelque sorte un absolu, elle n'est pas «l'Absolu» ultime; la communauté, entendue comme «une intégration de personnes dans l'entière sauvegarde de la vocation de chacune», est pour lui «une réalité, donc une valeur, à une approximation près, aussi fondamentale que la personne ${ }^{41}$ ». La liberté de Mounier est donc une liberté «sous conditions ${ }^{42} \gg$; son exercice dépend à la fois des limites imposées par la matérialité de la personne et de la liberté de toutes les personnes formant la communauté.

Pour Maritain, il va de soi pour une personne humaine de vivre en société pour atteindre le plein potentiel terrestre de sa personne spirituelle et de son individualité matérielle. L'homme a non seulement besoin de l'aide de son semblable pour satisfaire ses besoins de pain, d'abri et de vêtement, mais «aussi et avant tout de l'aide pour faire œuvre de raison et de vertu ${ }^{43} \gg$.

Pour que l'ensemble de la communauté puisse prospérer, l'individu est et doit être, pour Maritain, «inférieur au tout et subordonné au tout et doit servir comme organe du tout à l'œuvre commune ${ }^{44}{ }_{\gg}$. La vie en société ne doit toutefois pas grever toute liberté humaine. Au contraire, l'insertion de la personne dans sa communauté naturelle vient assurer la liberté et l'accomplissement temporel de la personne. Le bien commun doit être assuré non pas par des moyens répressifs de contrôle social, mais par une adhésion toujours répétée aux valeurs fondamentales de la communauté. Ainsi, selon Maritain, toute régulation externe des citoyens est vaine si elle n'a pas pour fin de développer dans la personne le sens de sa responsabilité créatrice et le sens de la communion: «se sentir responsable de ses frères ne diminue pas la liberté (mais) lui donne un poids plus lourd ${ }^{45}$.»

Tant pour la CTCC-CSN que pour Maritain et Mounier, cette vision communautaire prend sa source dans la tradition chrétienne et

41. Emmanuel Mounier, «Révolution personnaliste et communautaire», Euvres (Paris, Seuil, 1961), I: 175 .

42. Idem, «Le personnalisme...», 477.

43. Maritain, «La personne et le bien commun...», 198.

44. Ibid., 241.

45. Jacques Maritain, «Humanisme intégral», CEuvres complètes (Fribourg, Éditions universitaires, 1984), VI: 481 . 
en premier lieu dans la figure du saint. Selon la doctrine catholique, l'idéal communautaire du croyant est une société où règne le principe de mutualité de la personne (la communion des saints du credo), c'està-dire:

[...] l'union mutuelle que la charité établit entre les membres vivants de l'Église, les bienheureux dans le ciel et les âmes souffrantes du purgatoire. (Cette union) fait que sur la terre, les membres vivants de l'Église s'assistent mutuellement par leurs prières et leurs bonnes œuvres et sont aidés par l'intercession des saints du $\operatorname{ciel}^{46}$.

Le saint, tout en consacrant chaque action à Dieu, a la mission humanitaire d'aider la personne à gagner son salut. La communauté idéale n'est donc possible que lorsqu'en tant que chrétiens, les personnes sont responsables les unes des autres et vivent l'amour du prochain.

Le second principe est celui de la générosité de la personne telle qu'exprimée par la vertu théologale de la charité, définie dans le catéchisme comme la «vertu par laquelle la personne aime Dieu pardessus toutes choses, et son prochain (c'est-à-dire tous les hommes, même ses ennemis) comme elle-même pour l'amour de Dieu ${ }^{47}{ }^{7}$. Cet amour doit faire en sorte que chaque personne oriente ses actions afin de respecter et aimer l'autre, tant au niveau spirituel que physique. La charité telle qu'entendue par les catholiques de gauche ne se limite toutefois pas à son acceptation stricte du don individuel et ponctuel. La charité individuelle doit se traduire dans la Cité démocratique par la notion de justice sociale, qu'on associe au désir de la personne de s'accorder au bien commun.

\section{4 - LA DÉMOCRATIE}

Traditionnellement, les intellectuels catholiques québécois ont critiqué sévèrement l'État libéral canadien et le parlementarisme britannique. Ce système politique, implanté par des Anglo-Saxons, est considéré comme incompatible avec les mœurs et la culture de la société canadienne-française. La théorie de la souveraineté populaire est nécessairement erronée puisque l'autorité et le pouvoir sont issus de Dieu et non du peuple. De plus, la démocratie favorise un esprit individualiste qui peut s'opposer à leurs idéaux d'avancement de la nation canadienne-française. Ainsi, les catholiques de droite, comme

46. Le catéchisme des provinces ecclésiastiques de Québec, Montréal et Ottawa (Québec, 1944), 83-84.

47. Ibid., 24. 
les collaborateurs du journal L'Action catholique pendant les années trente, sont sympathiques à l'idéal d'une dictature éclairée par le clergé. Mais comme l'Église prêche le respect de l'ordre établi, ils ont généralement limité leur action politique au Québec à une simple critique des politiciens en place ${ }^{48}$.

Les humanistes-libéraux de la CTCC-CSN sont quant à eux résolument démocrates. Ils ne considèrent pas qu'ils doivent accorder leur foi aux idéaux démocratiques, mais adhèrent à l'opinion de Jacques Maritain, pour qui la démocratie «est liée au christianisme et que la poussée démocratique a surgi dans l'histoire humaine comme une manifestation temporelle de l'inspiration évangélique ${ }^{49}{ }^{\gg}$. Selon lui, les valeurs à la base du système démocratique, c'est-à-dire la foi dans la dignité de la personne et de l'humanité, dans les droits humains et dans la justice ${ }^{50}$, sont des valeurs essentiellement spirituelles héritées de la tradition chrétienne.

La CTCC-CSN adhère à cette analyse et estime que la démocratie ne peut survivre si elle n'est pas constamment nourrie par les principes chrétiens. Pour l'aumônier général Henri Pichette, «ce n'est pas par goût d'être à la mode que l'Église revendique présentement avec tant d'énergie la liberté des hommes. Elle ne fait que continuer à rappeler à l'humanité ce qui constitue un trait essentiel du christianisme ${ }^{51}{ }^{\prime}$. Bref, pour la centrale, seule la démocratie peut réaliser ou du moins s'approcher de l'idéal communautaire qui réalise la synthèse entre la liberté personnelle et le bien commun.

Comme nous l'avons mentionné précédemment, l'Église a été traditionnellement méfiante envers la démocratie, parce que toute autorité émane de Dieu et non des hommes ${ }^{52}$. Les catholiques de gauche de la CTCC-CSN rejettent eux aussi le contrat social rousseauiste et admettent l'existence d'une autorité politique naturelle. Ils l'interprètent toutefois dans le sens démocratique de la souveraineté populaire. Ainsi, Jean Marchand, le secrétaire général de la CTCC, écrit: «Le respect des pouvoirs établis n'exclut pas le droit de critique et d'amélioration. En effet, en régime démocratique, plus qu'en tout autre régime politique, les citoyens ont le droit et un devoir spécial de

48. Richard Jones, L'idéologie de L'Action catholique (1917-1939) (Québec, Presses de l'Université Laval, 1974), 201.

49. Jacques Maritain, «Christianisme et démocratie», Euvres complètes (Fribourg, Éditions universitaires, 1988), VII: 733.

50. Ibid., 737.

51. Henri Pichette, «Allocution de l'aumônier général», Procès-verbal de la trenteseptième session du congrès de la CTCC (Montréal, 1958), 281.

52. Jones, op.cit., 181-182. 
participer, de quelque manière, à l'administration de la chose publique $^{53}$.»

Cette interprétation de l'autorité est inspirée du personnalisme français. Selon Emmanuel Mounier, il est important de distinguer le pouvoir, qui est la force par laquelle on peut obliger autrui à obéir, et l'autorité qui est le droit de diriger et de commander. Si l'autorité requiert le pouvoir, le pouvoir sans autorité dégénère en puissance, c'est-à-dire que le droit cède la place aux simples rapports de force ${ }^{54}$.

Si les catholiques de gauche français acceptent l'origine divine de l'autorité, ils estiment que nul homme ou groupe particulier d'hommes en est le dépositaire terrestre absolu. Selon Jacques Maritain, l'autorité doit obligatoirement s'exercer dans le but de promouvoir le bien commun de la multitude. Pour lui, «le peuple est la substance même, la libre et vivante substance du corps politique ${ }^{55} 》$. Il possède son droit à se gouverner lui-même d'une manière permanente et n'aliène pas son autorité, reçue de Dieu, en l'abandonnant au pouvoir constitué. De l'autre côté, les élus reçoivent du peuple le droit de gouverner, de sorte que le peuple, par l'élection de ses représentants, restreint librement l'exercice de son droit à se gouverner dont il conserve la possession $^{56}$. Bref, comme l'a écrit Henry Bars à propos de Maritain, c'est comme vicaires, ou «représentants de la multitude» que les détenteurs de l'autorité dirigent la multitude, et c'est vers le bien commun de la multitude qu'ils doivent la diriger ${ }^{57}$.

Mounier résume cette thèse en posant comme principe que la personne, par sa dignité absolue, ne peut être soumise à moins qu'ellemême, que ce soit à un appareil social ou à un système politique. Pour lui, l'autorité est:

Au service de la liberté ontologique des personnes. [...] Le pouvoir a pour fin le bien commun qui n'est pas la somme des intérêts individuels [...] mais ce bien commun ne peut écraser une seule personne comme telle, refuser place à un seul acte d'authentique liberté spirituelle. Surtout, il ne peut pas se substituer aux personnes pour les décisions dont dépend leur destin même ${ }^{58}$.

53. Jean Marchand, «Rapport du bureau confédéral», Procès-verbal de la vingt-neuvième session du congrès de la CTCC (Sherbrooke, 1950), 53.

54. Emmanuel Mounier, «Manifeste au service du personnalisme», ELuvres, I: 623.

55. Maritain, «L'homme et l'État...», 509.

56. Henry Bars, La politique selon Jacques Maritain (Paris, Éditions ouvrières, 1961), 112.

57. Maritain, «Christianisme et démocratie...», 730-731.

58. Emmanuel Mounier, «Anarchie et personnalisme», Euvres, I: 677. 
La personne doit donc respecter l'autorité de façon absolue, mais pas nécessairement l'ordre établi. Elle doit savoir se plier devant l'autorité seulement s'il s'agit d'une véritable autorité démocratique librement acceptée. Selon Maritain, l'autorité doit être obéie «en conscience», c'est-à-dire «à la manière dont les hommes libres obéissent, dans l'intérêt du bien commun». Le respect de l'autorité est directement lié à la notion de justice: pour lui, «une autorité injuste n'est pas une autorité, pas plus qu'une loi injuste est une $\operatorname{loi}^{5{ }^{59}}{ }$.

Cette conception de l'autorité implique donc que la personne, en plus de jouir de libertés d'autodétermination inaliénables, qu'elle exerce grâce au suffrage universel, a la responsabilité de veiller au bon exercice de l'autorité pour ainsi promouvoir le bien commun.

Bien que tous les catholiques de gauche français soient démocrates, tous ne s'entendent pas sur le type de système démocratique qui est le plus apte à réaliser leur idéal communautaire et personnaliste.

Le régime politique idéal tel que conçu par Emmanuel Mounier, est une démocratie décentralisée ayant une certaine parenté avec la démocratie directe et autogestionnaire des anarchistes proudhoniens ${ }^{60}$. Il prône une décentralisation effective du pouvoir exécutif et législatif à tous les niveaux et dans tous les domaines. Une «articulation horizontale» des municipalités serait établie pour coordonner les politiques au niveau national, établissant ainsi un genre de confédération de communes autonomes ${ }^{61}$. L'État conserve uniquement dans ce système la responsabilité d'arbitrer les conflits entre les différentes communautés. En cas d'impasse, Mounier prévoit l'usage du référendum d'initiative populaire, qu'il oppose au plébicite, d'initiative d'État ${ }^{62}$.

Le modèle politique adopté par Maritain ressemble, quant à lui, davantage aux démocraties parlementaires libérales. En plus du suffrage universel, il prône un régime représentatif où les pouvoirs législatifs et exécutifs sont séparés et où les fonctions de l'exécutif sont «exercées par des organes gouvernementaux émanant de la multitude par suffrage indirect, et responsables devant l'organe recteur suprême élu directement par le peuple ${ }^{63} »$.

59. Maritain, «L'homme et l'État...», 629.

60. Marie-Thérèse Collot-Guyer, La cité personnaliste d'Emmanuel Mounier (Nancy, Presses universitaires de Nancy, 1983), 208-209.

61. Ibid., 249.

62. Ibid., 246.

63. Maritain, «Humanisme intégral...», 488-489. 
À l'instar de Maritain, la CTCC-CSN adopte clairement la démocratie libérale comme idéal politique. Pour la centrale, la meilleure tradition démocratique «se trouve dans le parlementarisme d'inspiration britannique dont un caprice de l'Histoire nous a rendus tributaires. Cette pensée est riche, exhaustive, presque millénaire ${ }^{64} \gg$. Bien que les militants de la centrale soient remplis d'admiration pour la figure intellectuelle d'Emmanuel Mounier, son engagement révolutionnaire ainsi que son modèle de démocratie directe et décentralisée sont étrangers à leur culture politique. La «véritable démocratie politique» qu'ils opposent au régime de Duplessis est fondamentalement le parlementarisme britannique prévu par la constitution canadienne. On retrouve d'ailleurs, dans la déclaration adoptée par la centrale en 1960, les grands principes qui se trouvent à la base de cette tradition: «La CSN a foi dans la vraie démocratie politique, parce que c'est le système qui garantit le mieux la liberté des citoyens et leur participation aux responsabilités civiles. La démocratie implique le suffrage universel et la division des pouvoirs entre le législatif, l'exécutif et le judiciaire ${ }^{65} . \gg$ Les revendications politiques formulées par la centrale pendant toute la période qui nous concerne se placent donc dans une perspective résolument démocratique et réformiste. Ainsi, lors des élections provinciales de 1960, la centrale ne réclame pas de changements profonds au régime politique mais plutôt des mesures visant à en faciliter la bonne marche: publication d'un compte rendu des débats de l'Assemblée et du Conseil législatif, droit de vote à dix-huit ans, réforme de la carte électorale et loi limitant les dépenses électorales des candidats ${ }^{66}$.

\section{5 - LE CHRÉTIEN, L'ÉGLISE ET LA POLITIQUe}

En faisant la promotion d'une démocratie inspirée et fondée sur des principes chrétiens, les militants humanistes-libéraux de la CTCCCSN ont été confrontés au problème de l'implication de la religion dans le domaine temporel. Fondée dans la mouvance de l'action catholique spécialisée, la centrale reste fidèle au principe de l'implication des croyants et de la religion dans les affaires de la Cité. Comme le fait valoir l'aumônier du Conseil central de Montréal de la CSN, le chrétien doit «être présent au monde aussi profondément que l'âme est insérée dans le corps. C'est-à-dire que le chrétien doit prendre au sérieux les choses de ce monde ${ }^{67}{ }$. 1960,2

64. Paul Nogaret, «Pourquoi le Canada n'a pas son drapeau?», Le Travail, 4 mars

65. CSN, «Déclaration de principes de 1960..», 130.

66. «Nos demandes et leurs programmes», Le Travail, 10 juin 1960, 3.

67. Lafontaine, «Le christianisme...», 7. 
Sous l'influence dles penseurs catholiques de gauche, la CTCCCSN en vient toutefois à rejeter l'intervention directe de l'Église et du clergé dans le domaine temporel et à dénoncer chez l'Église ce que même l'aumônier général de la centrale qualifie de «désir de domination, d'intrusion ou de théocratie, où serait sacrifiée l'autonomie légitime d'un domaine profane ${ }^{68}$.

Le premier principe qui guide cette prise de position est celui de la distinction et de la primauté du domaine spirituel sur le domaine temporel. Selon Maritain, c'est le christianisme qui a été historiquement l'initiateur de cette notion. La société antique était selon lui «hiéropolitique», le corps politique et le corps religieux étant invariablement le même ${ }^{69}$. Lorsque Jésus parla des «choses de César et des choses de Dieu», il établit la distinction entre les deux domaines tout en posant l'absolue primauté du spirituel:

Par son enseignement général sur le Christ et sur la Rédemption, comme par ses précisions sur le gouvernement civil et l'Église, il nous fait voir dans toute sa force la suprématie de celle-ci. Par sa doctrine de la nature et de la grâce, et de la subordination des fins, il nous fait comprendre la primauté des fins spirituelles sur les fins politiques, et du domaine universel de la grâce sur toutes les partialités de la nature. Par sa doctrine de la vie humaine et des vertus, il met en lumière le primat de la contemplation infuse, propre aux esprits élevés par la grâce au partage de la vie divine, sur l'action au dehors, commune [...] aux corps et aux esprits ${ }^{70}$.

Malgré la distinction et la primauté du domaine spirituel, le domaine temporel n'en est pas séparé ou «désaccordé» mais plutôt en connexion avec lui et sous son ordination. En découlent pour Maritain trois grands principes: la liberté de l'Église face à l'État; la supériorité de l'Église sur l'État, de la même façon que le royaume céleste est audessus du royaume périssable; la coopération indirecte entre l'Église et l'État ${ }^{71}$. L'application de ces principes dans une société moderne et démocratique exige que l'Église jouisse d'une totale indépendance face à l'État, que Maritain qualifie de «cité laïque vitalement chrétienne» ou «d'État laïque chrétiennement constitué ${ }^{72} »$.

68. Henri Pichette, «Allocution de l'aumônier général», Procès-verbal de la trentequatrième session du congrès de la CTCC (Québec, 1955), 306.

69. Jacques Maritain, «Pour une philosophie de l'histoire», Euvres complètes (Fribourg, Éditions universitaires, 1985), X: 709.

70. Idem, «Primauté du spirituel», Euvres complètes (Fribourg, Éditions universitaires, 1984), III: 865 .

71. Idem, «Humanisme intégral...», 475.

72. Ibid., 491. 
Mais si l'Église s'implique directement dans les affaires publiques en se servant instrumentalement de l'État et si elle entretient des liens organiques avec lui, elle risque de sombrer avec lui s'il est discrédité ou renversé par une révolution. Il est donc impératif que l'Église exerce son autorité non pas sur l'État mais sur les personnes humaines et, par elles, sur la société qu'elles forment. Selon M ${ }^{\mathrm{gr}}$ Émile Guerry, président à la fin des années cinquante de la Commission épiscopale française du monde ouvrier, le chrétien a le droit et le devoir de travailler par tous les moyens légitimes à l'instauration d'un régime différent du régime capitaliste, pourvu que soient sauvegardés les principes du droit naturel conformément à la doctrine de l'Église. Selon lui, «l'Église n'est pas plus liée au capitalisme du XIX ${ }^{\mathrm{c}}$ siècle ou du $X^{c}$ siècle qu'aux divers régimes qui se sont succédé depuis sa fondation $\left.{ }^{73}{ }\right)$.

L'Église, dépositaire de l'autorité spirituelle, doit donc remplir pleinement son rôle et ne pas s'embarrasser de questions qui ne la concernent pas directement et qui risquent d'entraver sa mission. Pour Mounier, il est essentiel «qu' on laisse donc le christianisme à sa tâche, qu'on parle à visage découvert au nom des jugements que l'on porte, et que l'on ne fasse pas à Dieu de faux papiers pour l'engager dans l'arène électorale ${ }^{74}$ ».

La séparation de l'Église et de l'État implique également la pluralité religieuse. Selon Maritain, la cité, bien qu'essentiellement chrétienne, doit accepter en son sein toutes les confessions. Ceci n'empêche pas les croyants et les non-croyants d'œuvrer de concert, puisque cette cité n'est pas «sacrale chrétienne» mais "profane chrétienne»; elle n'exige pas une profession de foi chrétienne mais une simple adhésion à ses principes de base, ce qui rend possible «le convivium de chrétiens et de non-chrétiens dans la cité temporelle ${ }^{75}$ ».

La liberté religieuse, liée à la liberté de choix fondamentale de la personne, est donc compatible avec la cité chrétienne. Le pluralisme demande toutefois l'intégration dans cette cité de confessions professant des valeurs contraires aux idéaux chrétiens. Pour Maritain, l'arme la plus efficace contre les «hérétiques» est de rendre leur action inefficace par la force de la vérité, en mettant de l'avant la philosophie chrétienne. La force de la démocratie chrétienne réside donc dans la foi qu'elle a en elle-même ${ }^{76}$ et dans son idéal, qui est «un rassem-

73. Émile Guerry, cité par Jean-Paul Lefebvre dans «La lutte des classes», Le Travail, 12 janvier 1960, 5 .

74. Emmanuel Mounier, «Feu la chrétienté», CEuvres, III: 541.

75. Maritain, «Humanisme intégral...», 522-523.

76. Bars, op. cit., 136. 
blement pluraliste de chrétiens et d'incroyants autour d'un idéal métaphysique et de directions d'engagements communes ${ }^{77}$ ».

D'autre part, pour les catholiques de gauche français, le pluralisme et la liberté religieuse ne sont possibles que si les laïcs peuvent jouir d'une indépendance d'action par rapport au clergé. Pour Emmanuel Mounier, l'engagement personnel du laïc est nécessaire d'autant plus que le besoin d'engagement, d'adhésion et de perpétuelle conversion de l'homme est une partie intégrante de la personne. Pour lui, la personne est en grande partie constituée par son adhésion à «une hiérarchie de valeurs librement adoptées, assimilées et vécues par un engagement responsable et une constante conversion; elle unifie ainsi toute son activité dans la liberté et développe par surcroît à coups d'actes créateurs, la singularité de sa vocation ${ }^{78}$ ». Pour s'accomplir pleinement en tant que personne, le chrétien doit donc s'engager, prendre position à part entière autant dans le domaine spirituel que dans le monde temporel.

Suivant l'analyse des catholiques de gauche français, la CTCCCSN arrive à la conclusion qu'il existe une véritable «vocation du laïc», différente de celle du clergé, qui consiste à faire pénétrer les principes du catholicisme dans leur vie quotidienne et celle de leurs pairs «par amour de leur mère l'Église, par esprit de respect au Pape et aux évêques choisis pour les guider dans leurs initiatives ${ }^{79}$ ».

Appliquant ses théories de séparation de l'Église et de l'État au niveau personnel, Jacques Maritain distingue deux façons, pour le chrétien, de s'engager dans les affaires de la cité civile ${ }^{80}$. En agissant en tant que chrétien, le chrétien (généralement membre du clergé) agit comme représentant de l'Église, fonde des institutions spécifiquement catholiques qui mettent en cause directement l'Église. Pour Maritain, ce mode d'action est approprié en ce qui concerne les questions purement spirituelles et les «questions mixtes», relevant à la fois du domaine temporel et spirituel (le mariage, les actes de décès, etc.).

Agir en tant que chrétien dans les domaines purement temporels risque toutefois, comme nous l'avons vu précédemment, de compromettre l'intégrité et le statut de l'Église, tout en menaçant sa mission spirituelle primordiale. Il est donc préférable d'agir en chrétien, c'està-dire comme membre d'une société pluraliste. Guidé par les principes chrétiens, le laïc participe et agit socialement en collaborant à des institutions séculières.

77. Mounier, «Révolution personnaliste...», 219.

78. Idem, «Manifeste au service du personnalisme», 523.

79. «Mêlez-vous de vos affaires», Le Travail, 27 août 1954, 3.

80. Maritain, «Humanisme intégral...», 617-634. 
Les humanistes libéraux de la CTCC adhèrent à ces principes et accordent une place primordiale à l'action des laïcs. Selon eux, c'est dans le domaine de la vie publique que les laïques ont «un rôle irremplaçable à jouer s'ils veulent sincèrement que s'instaure le règne social du Christ et que ses lois comme ses droits triomphent dans la vie politique, professionnelle, économique et sociale ${ }^{81} \gg$. Les membres du clergé gardent donc un rôle social: celui de «chiens de garde» des valeurs chrétiennes. C'est à eux qu'incombe la tâche de garder la morale catholique bien vivante auprès des laïcs, sans toutefois s'impliquer directement dans le domaine temporel.

Suivant ces principes, la confessionnalité des institutions socioéconomiques en général est une situation dangereuse puisqu'elle menace à long terme la présence de l'Église dans le domaine temporel. De plus, nier le rôle des laïcs, c'est refuser d'admettre que le Québec est devenu une société industrialisée et pluraliste. Pour les militants de la CTCC, la présence catholique dans la société ne doit pas être défensive et passéiste. Ils se font les promoteurs d'un catholicisme qui accepte pleinement la modernité, où les laïcs jouent le rôle primordial de toujours actualiser, dans son intégrité, le message du Christ. Ainsi, nombreux sont les militants qui estiment que: «Tel qu'il est, le monde moderne ne nous effraie pas, ne nous dégoûte pas; disons-le même hautement, il ne nous scandalise pas. Il est plein de magnifiques ressources qui peuvent servir au bien commun comme elles peuvent servir au mal; si elles servent au mal, ce n'est pas sa faute, c'est la nôtre ${ }^{82}$.»

En véhiculant une idéologie mettant de l'avant l'engagement personnel des laïcs et le pluralisme, la CTCC a ainsi fini par être confrontée au besoin de déconfessionnaliser ses structures. En limitant le droit d'adhérer aux seuls catholiques, la centrale entre en contradiction avec ses principes de liberté de choix et d'engagement personnel. Les militants humanistes-libéraux en sont donc venus à la conclusion que la foi est une question qui relève uniquement de la personne elle-même. Après la déconfessionnalisation de 1960, la centrale cesse donc d'exiger l'adhésion de ses membres à la foi catholique, mais seulement aux grands principes chrétiens de l'humanisme libéral, tels qu'énoncés dans sa déclaration de principes.

Bien qu'elle ait été motivée essentiellement pour des raisons idéologiques, il faut toutefois noter que la déconfessionnalisation a aussi été motivée par des questions de pertes d'effectifs. Les dirigeants de

81. «Au laïcisme, opposons le laïcat», Le Travail, 31 juillet 1953, 2.

82. «Mêlez-vous de vos affaires», Le Travail, 27 août 1954, 3. 
la CTCC en viennent à la conclusion, à la fin des années cinquante, que l'orientation confessionnelle a empêché la centrale de bénéficier de la période de croissance qu'a connue le mouvement syndical dans l'après-guerre. Le résultat net semble leur avoir donné en partie raison puisque la centrale met fin, l'année même de la déconfessionnalisation, à une période de stagnation de ses effectifs, qui aura duré douze ans ${ }^{83}$.

\section{6 - LE NATIONALISME}

La vie politique québécoise est, depuis la Confédération, fortement marquée par la question nationale. En formulant des revendications sur la scène politique, la CTCC-CSN n'a pu éviter de prendre position face au nationalisme canadien, canadien-français et québécois.

Confrontés au «nationalisme intégral» des catholiques conservateurs de L'Action française, Maritain et Mounier se montrent très critiques envers cette idéologie. Pour Maritain, le nationalisme a mené à la réaction anti-démocratique du $\mathrm{XX}^{\mathrm{e}}$ siècle. C'est «une divinité terrestre dont l'égoïsme absolu est sacré ${ }^{84} »$, un paganisme anti-chrétien. Mounier, quant à lui, ne rejette pas a priori le patriotisme mais le subordonne aux intérêts du genre humain:

Il est superflu de rappeler ici les conditions réitérées de ces deux abstractions: nationalisme et internationalisme, et de la variété raciste du nationalisme. Elles consacrent la patrie comme personne morale régie par la charité, elles condamnent l'individualisme national qui se ferme à la charité en se repliant sur son égoïsme groupa ${ }^{85}$.

Si le patriotisme a une certaine valeur positive aux yeux de Mounier, le nationalisme représente pour lui un sentiment néfaste, une corruption du légitime désir d'appartenance de la personne à sa communauté naturelle ${ }^{86}$. De par sa nature, l'idéologie nationale est une mystique qui met en danger la nature propre de chaque individu. Selon

83. Il est intéressant de noter que les syndicats catholiques québécois ont précédé les syndicats catholiques français sur la voie de la déconfessionnalisation. Ce n'est qu'en 1964 que la CFTC devient la Confédération française démocratique du travail (CFDT), soit quatre ans après la CTCC.

84. Maritain, «L'homme et l'État...», 488.

85. Emmanuel Mounier, «Les chrétiens devant le problème de la paix», Euvres, I: 825

86. «Le nationalisme se sert du patriotisme comme le capitalisme se sert du sentiment naturel de la propriété personnelle, afin de donner [...] à un égoïsme collectif un aliment sentimental en même temps qu'une justification morale» (Mounier, «Manifeste au service...», $627)$. 
lui, toute idéologie qui place la nation au rang «d'unité sociale suprême, source et terme de tous droits et devoirs, mérite d'être réprouvée comme contraire à la dignité de la personne humaine ${ }^{87}$ ». Mounier va même plus loin en prétendant que le nationalisme est, comme le capitalisme, un surgeon de l'esprit bourgeois, du «désordre établi». Pour lui, le patriotisme est essentiellement un sentiment issu du peuple, alors que le nationalisme «descend de l'État sur les personnes [...] Il se sert du patriotisme comme le capitalisme se sert du sentiment naturel de la propriété personnelle ${ }^{88} \gg$.

En ce qui concerne le nationalisme, les dirigeants de la CTCCCSN reprennent en grande partie l'analyse de Mounier. Désirant affranchir la centrale de l'étiquette nationaliste, Jean Marchand déclare que la centrale "s'est toujours refusée à devenir une organisation à l'usage exclusif des travailleurs canadiens-français ${ }^{89}{ }{ }^{\prime}$. Le nationalisme apparaît comme une idéologie qui désolidarise les travailleurs, qui va à l'encontre de la démarche syndicale. C'est un leurre, comme un tour de passe-passe utilisé par les bourgeois pour détourner la classe ouvrière de ses vrais problèmes (justice sociale, démocratie, droits et libertés, humanisation de l'économie...). Les catholiques de gauche de la centrale s'emploient donc à dénoncer «'imposture ${ }^{90} »$.

Outre l'influence française, l'antinationalisme de l'humanisme libéral est en grande partie un produit de l'époque (l'après-guerre) et du milieu (le Québec de Duplessis) d'où il est issu. Le nationalisme, élément clé du fascisme et du national-socialisme, se retrouve disgracié après la guerre au même titre que les valeurs autoritaires qu'ils véhiculaient. De plus, la lutte des catholiques de gauche au Québec contre le conservatisme de l'Union nationale a imprimé à la longue, dans l'esprit des militants, l'équation nationa-

87. Ibid., 825 .

88. Mounier, «Manifeste au service...», 828 .

89. Jean Marchand, «Rapport du bureau confédéral», Procès-verbal de la trenteneuvième session du congrès de la CSN (Montréal, 1960), 104.

90. «Sous le couvert du patriotisme, la bourgeoisie canadienne-française s'opposa au développement $(\mathrm{du})$ mouvement syndical. C'était une façon de nier l'existence de problèmes nouveaux et croissants qu'elle se refusait à prendre en considération. Composée d'intellectuels formés à l'école du libéralisme du siècle dernier, elle était trop préoccupée de conserver, par exemple, le code civil français, pour penser aux besoins d'une législation sociale favorable à la promotion collective (des travailleurs) [...] Elle préféra faire servir ses talents oratoires à des campagnes politiques retentissantes, couronnées de certains succès d'ordre politique ou culturel, mais qui restèrent le plus souvent vides de sens pour les travailleurs. Dans les usines, les ouvriers avaient le droit de parler français, mais on ne les comprenait pas, ou on ne les écoutait pas» (Fernand Jolicoeur, "Les problèmes ouvriers du Québec», Chronique sociale de France, 65,5 (septembre 1957): 434). Fernand Jolicoeur est à l'époque le directeur du service de l'éducation de la CTCC. 
liste $=$ réactionnaire. Ainsi, comme Duplessis s'est fait le champion de l'autonomie provinciale, cette doctrine prend automatiquement un sens ignominieux: «Pour un bon nombre de travailleurs, l'autonomie provinciale évoque l'idée de matraque. L'on aura beau sonner le ralliement autour du fleurdelisé, les travailleurs ne peuvent oublier qu'il est déjà taché du sang de plusieurs de leurs camarades ${ }^{91}$.»

Il en est de même, au début des années soixante, pour le projet indépendantiste alors en élaboration, qui revêt pour les dirigeants de la CTCC-CSN les mêmes attributs autoritaires et réactionnaires. Jean Marchand s'explique: «On ne s'est pas suffisamment posé la question de savoir si un Québec indépendant du reste du Canada serait nécessairement la terre d'élection de la justice sociale et du respect intégral des droits de l'homme et du citoyen ${ }^{92}$.» De façon générale, les humanistes libéraux semblent douter de la capacité du Canada français d'assumer la pleine responsabilité d'un État moderne. Suivant l'opinion de Maritain, qui se dit en complet désaccord avec «le soi-disant principe des nationalités compris dans le sens que chaque groupe national doit se constituer en Etat séparé93 ${ }^{93}$, ils croient que pour leur propre bien-être, les Canadiens français ont intérêt à rester dans le giron de la fédération canadienne. Leur obsession nationale, c'est-àdire la promotion indue des caractères ethniques de la nation (langue, religion, coutumes, etc.), a depuis toujours endigué ses énergies créatrices et entretenu le peuple dans le chauvinisme et l'ignorance. En résultent une incapacité culturelle et un narcissisme qui rendent impossible un Québec indépendant viable. Bref, ce n'est pas par l'indépendance du Québec que la classe ouvrière canadienne-française pourra être promue, car «ce n'est pas en restant ignorants et retardataires que les Canadiens français pourront se tailler la place qui leur est due ${ }^{94} \gg$.

Toutefois, l'antinationalisme de la centrale n'est véritablement qu'une dénonciation du nationalisme traditionnel et de l'indépendantisme. Il ne signifie pas que l'humanisme libéral de la CTCC-CSN soit exempt de toute référence nationale positive. Bien au contraire, la centrale adopte à la même époque un nationalisme canadien-français dont les axes théoriques sont le bilinguisme, l'antiaméricanisme et le fédéralisme.

91. Gérard Picard, «Rapport moral du président général de la CTCC», Procès-verbal de la trente-deuxième session du congrès de la CTCC (Québec, 1953), 36.

92. Marchand, «La CSN et le séparatisme», Le Travail, décembre 1961, 5.

93. Maritain, «L'homme et l'État...», 488.

94. Jean Marchand, «L'instruction gratuite: une nécessité chez nous», Le Travail, 21 novembre $1958,4$. 
Dans les faits, le nationalisme de la CTCC-CSN ne change pas beaucoup avec l'humanisme libéral. Il reste pancanadien, fait la promotion d'un Canada indépendant (davantage des États-Unis que de la Grande-Bretagne) et se porte toujours à la défense des Canadiens français ${ }^{95}$. La grande différence réside essentiellement dans l'abandon des thèmes conservateurs (passéisme, messianisme, agriculturisme, etc.). L'antinationalisme affiché par la CTCC-CSN est donc en réalité un rejet des «abus» du nationalisme. Les militants de la CTCC-CSN opposent ainsi leur «sain patriotisme», qui prône l'adhésion et l'identification à un État de droit respectueux des droits de la personne et des valeurs démocratiques, au nationalisme traditionnel et au néonationalisme québécois dont les tendances «tribales» semblent suspectes.

\section{CONCLUSION}

Pendant les années cinquante, la pensée politique de la CTCCCSN adopte la plupart des grands thèmes politiques élaborés par les penseurs catholiques de gauche. On retrouve, énoncés pour la plupart dans les deux déclarations de principes de la centrale, les mêmes postulats que chez Emmanuel Mounier et Jacques Maritain. Ainsi, la personne humaine, unité fondamentale de la société, est considérée comme un être autonome et libre, dont les droits et la liberté ne peuvent être brimés par une quelconque tyrannie collective.

De plus, l'autorité politique n'est plus considérée, comme c'était le cas chez les catholiques conservateurs, comme un absolu dont le respect est obligatoire en toutes circonstances. Comme le peuple est le seul dépositaire de l'autorité temporelle de Dieu, il jouit d'un droit inaliénable de contester et même de révoquer tout dirigeant, dont le rôle politique se limite d'ailleurs à celui de «vicaire de la multitude». La CTCC-CSN adhère donc pour l'essentiel aux grandes institutions de la démocratie libérale: droits individuels, suffrage universel, parlementarisme.

L'influence des philosophes catholiques de gauche français se fait également sentir lorsque la centrale définit la place que doit occuper l'Église dans la société civile. C'est essentiellement la tâche du laïc de faire rayonner les principes chrétiens sur les plans politique et temporel, le clergé restant confiné au domaine spirituel, jouant le rôle de protecteur de l'intégrité du message de l'Église.

95. Louis-Marie Tremblay, Le syndicalisme québécois (Montréal, Presses de l'Université de Montréal, 1972), 41-42. 
Pour ce qui est de la question nationale, la CTCC-CSN hérite, du moins dans son discours, de l'antinationalisme de Jacques Maritain et d'Emmanuel Mounier. Cette attitude est particulièrement évidente lorsque les dirigeants de la centrale fustigent le mouvement indépendantiste québécois naissant. La CTCC-CSN continue toutefois dans les faits de faire la promotion d'un nationalisme canadien-français dont les caractéristiques sont l'antiaméricanisme, la défense du bilinguisme et l'adhésion au fédéralisme. Les militants se contentent donc d'évacuer les thèmes conservateurs de leur nationalisme tels que le concept de race canadienne-française et le messianisme religieux.

Au regard de la pensée politique, il appert que ce sont les idées de Jacques Maritain et du courant démocrate-chrétien qui ont le plus d'affinités avec celles de la CTCC-CSN. Ceci ne signifie pas que les personnalistes et Emmanuel Mounier n'ont pas eu d'impact sur cet aspect de l'idéologie de la centrale. Bien au contraire: on retrouve, exprimés dans les deux déclarations de principes de la CTCC-CSN, l'essentiel des postulats de Mounier concernant la personne et son intégration dans la communauté. Ce sont ses conclusions pratiques, relativemente au régime politique à adopter (démocratie décentralisée, limitation du pouvoir de l'État, importance du plébiscite, etc...) qui sont étrangères à la culture politique des militants de la centrale. 